\title{
GDAS atmospheric models in astroparticle shower simulations
}

\section{J. Grisales-Casadiegos, ${ }^{a, *}$ C. Sarmiento-Cano ${ }^{b, c}$ and Luis A. Núñez ${ }^{a, d}$ on behalf of the LAGO Collaboration \\ (a complete list of authors can be found at the end of the proceedings)}

${ }^{a}$ Escuela de Física, Universidad Industrial de Santander, Bucaramanga, Colombia

${ }^{b}$ Instituto en Tecnologías de Detección y Astropartículas, Centro Atómico Constituyentes, Buenos Aires, Argentina

${ }^{c}$ Consejo Nacional de Investigaciones Científicas y Técnicas (CONICET), Buenos Aires, Argentina

${ }^{d}$ Departamento de Física, Universidad de Los Andes, Mérida, Venezuela

E-mail: jennifer.grisales@saber.uis.edu.co,

christian.sarmiento@iteda.cnea.gov.ar, lnunez@uis.edu.co

\begin{abstract}
Atmospheric conditions affect the development of cascades of secondary particles produced by primary cosmic rays. Global Data Assimilation System, implementing atmospheric models based on meteorological measurements and numerical weather predictions, could significantly improve the outcomes of the simulations for extensive air showers.

In this work, we present a methodology to simulate the effect of the atmospheric models in secondary particle flux at the Earth's surface. The method was implemented for BucaramangaColombia, using ARTI: a complete computational framework developed by the Latin American Giant Observatory Collaboration to estimate the particle spectra on Water Cherenkov Detectors depending on the geographical coordinates. We observe differences in the total flux that varies monthly concerning the subtropical summer atmospheric profile as preliminary results.
\end{abstract}

$37^{\text {th }}$ International Cosmic Ray Conference (ICRC 2021)

July 12th - 23rd, 2021

Online - Berlin, Germany

\footnotetext{
*Presenter
} 


\section{Introduction}

Cosmic ray primaries, i.e., particles impinging the upper atmosphere with energies below $10^{15} \mathrm{eV}$, are modulated by the solar activity[1]. They interact with the geomagnetic fields, drafting a new discipline: Space Weather Physics. The interaction of these cosmic rays with the nuclei of atomic elements in the atmosphere produces extensive air showers (EAS), resulting from the complex convolution of physical phenomena when particles travel through the atmosphere.

Understanding the atmosphere is crucial for any cosmic ray observatory, e.g. in the LAGO collaboration, the network of detectors spans through different geographic sites, covering a broad range of geomagnetic rigidity cutoffs and atmospheric depths. The LAGO collaboration exploits its array of detectors to examine the influence of space weather on the cosmic ray flux at the Earth surface. To this end, LAGO has developed a precise simulation scheme considering three factors with different spatial and temporal scales: Geomagnetic effects; development of EAS through the atmosphere and the response of ground-level detectors [2].

In this work, we use GDASTOOL: a python routine based on the Global Data Assimilation System (GDAS) [3] to illustrate the significant impact of detailed atmospheric and climate models have on the cosmic ray flux at the Earth's surface. This routine is available in the latest versions of CORSIKA ${ }^{1}$ (Cosmic Ray Simulations for KAskade [4]) that allows us to obtain a specific atmosphere model for a particular daytime and geographic site.

We present a methodology to create monthly atmospheric profiles for any location. Moreover, as a preliminary result, we had estimated the influence of these monthly atmospheric models on the secondary particle flux that reaches the LAGO WCD detectors.

\section{Atmospheric models for the background flux study}

Density is one of the most critical atmospheric parameters, influencing the probability of particle interaction as the EAS evolves. This is concentrated in the first $30 \mathrm{~km}$ from the ground, decreasing as altitude increases and varies by weather changes.

The density is modelled by the vertical atmospheric depth [5], measured in $\mathrm{g} / \mathrm{cm}^{2}$ and defined as

$$
X_{h}=\int_{h}^{\infty} \rho\left(h^{\prime}\right) d h^{\prime},
$$

where $\rho(h)$ is the density as a function of height, $h$, above the Earth.

For the EAS simulations, we use CORSIKA, which implements a Monte Carlo scheme to recreate their propagation when initiated by protons, photons, nuclei, or any other impinging particle [4]. Also, it models the atmosphere through different types of configurations with a certain level of detail. CORSIKA considers atmosphere made by five density layers, where the first four can be approximated by equations 2 y 3 :

$$
X_{h}=a_{i}+b_{i} e^{\frac{-h}{c_{i}}} \quad i=1, \ldots, 4,
$$

while in the highest layer, the vertical atmospheric depth decreases linearly with height as:

$$
X_{h}=a_{5}-b_{5} \frac{h}{c_{5}} \quad \text { with } h_{\max }=112.8 \mathrm{~km} .
$$

${ }^{1}$ CORSIKA has gdastool since version 76300 
In the above expressions, $a_{i}, b_{i}, c_{i}$, are the corresponding parameters of each atmospheric layer, which should be continuous across the boundaries of the different segments [4].

CORSIKA allows configuring these parameters in three different ways: using the predefined models for specific locations, selecting climate regions, or manually entering the data to include more detailed parameters, which is the configuration used in this work.

To build the atmospheric profiles, we used the data from the Global Data Assimilation System (GDAS) [3], which produces realistic climate predictions, describing the state of the atmosphere for certain variables in time, latitude and altitude. At a given time, $t_{0}$, the observations provide a value for a weather variable (temperature, pressure, humidity). The analysis combines observation and prediction to improve the model at a particular time $t_{0}$, implementing a forecast for a later time $t_{1}$ [6]. Previous studies at the Pierre Auger Observatory have shown the advantage of GDAS-based atmospheric profiles for EAS reconstructions [6].

\subsection{Monthly atmospheric profiles}

GDASTOOL is a python routine that downloads the GDAS data, reads the binary file and extracts the altitude, temperature, pressure and relative humidity information for an exact time and a specific location $[4,6]$.

From this information, it calculates the air density, atmospheric depth, the parameters $a_{i}, b_{i}$, $c_{i}$ and the boundaries of one of the five layers of the atmosphere.

We are considering that at the local level, the estimation of atmospheric variables at different times of the day can change drastically. A single atmospheric profile such as those generated by GDASTOOL is not suitable for studying the secondary flux.

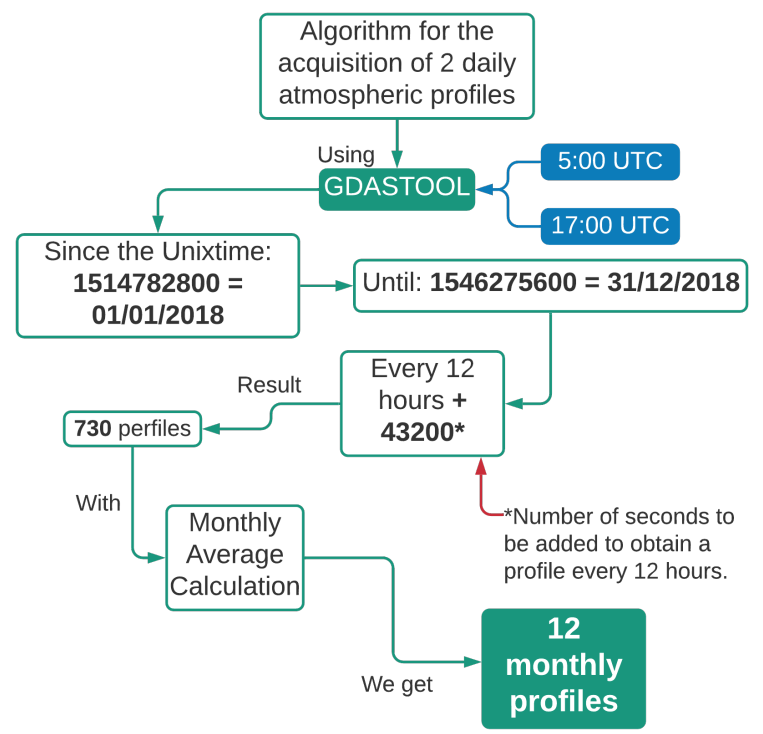

Figure 1: Logical sequence used to extract and to build the 12 month average profiles for the city of Bucaramanga for the year 2018.

For this work, we have defined the month as a time criterion. We implemented a computational algorithm using GDASTOOL that extracts data from two different times: 0:00h and 12:00 (UTC-5 local time), for all days of a year at any geographic position and build with these monthly average 
atmospheric profiles. For example, for January, we extracted 62 profiles for the year 2018. By averaging, we prevent our model falls into a local fluctuation. In our case, we show in fig. 1, the algorithm applied for the city of Bucaramanga-Colombia $\left(7.13^{\circ} \mathrm{N}, 73.00^{\circ} \mathrm{W}\right)$ where LAGO has a small array of WCDs.

We extracted 730 profiles per year, i.e. two profiles per day. Fig. 2 (left) shows all the instantaneous profiles specifically for January 2018 (solid line) and their average (dashed line). We performed the averaging between the parameters of the same atmospheric layers. Finally, we obtained the boundaries between layers and the parameters $a_{i}, b_{i}, c_{i}$ of each layer in each month.
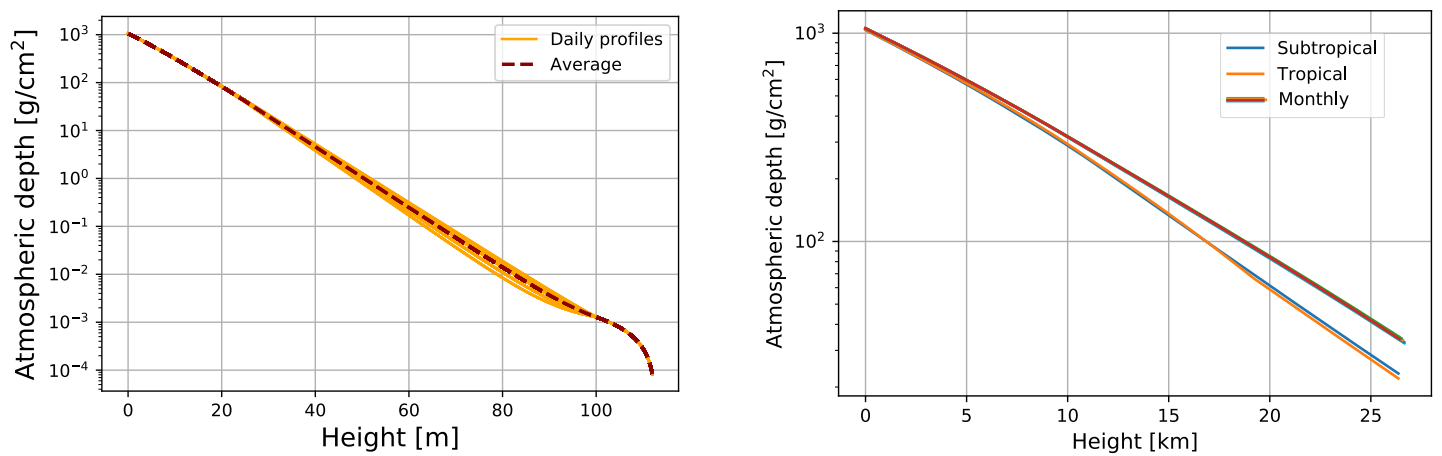

Figure 2: Left side shows in solid lines 62 density GDAS profiles for the month of January in Bucaramanga $\left(7.13^{\circ} \mathrm{N}, 73.00^{\circ} \mathrm{W}\right)$ and in dash line their average. Right side illustrates the first $30 \mathrm{~km}$ of the GDAS month density profiles for Bucaramanga and CORSIKA-Sub-tropical summer default profiles.

Thus, we built 12 monthly profiles for the year 2018 in Bucaramanga and compared them with the predetermined profiles for this location. We observed significant differences, as shown on the right side of fig. 2. Here, we plot the first $30 \mathrm{~km}$ from sea level, which accounts for most atmospheric matter density.

\section{Particle flux and atmospheric models}

We generate the most realistic primary flux for simulating each shower generated by the different impacting particles. To estimate this particle flux, we use ARTI [2] to establish the time required to integrate the flux at ground level. Finally, we defined the initial conditions for running each of these simulations. The initial conditions correspond to the geomagnetic field components, the height above sea level, the type of primaries, the angles of incidence of the primaries and the atmospheric model.

Figure 3 displays the spectrum of secondaries and the total secondary flux, using the constructed April atmospheric profile, and helps to understand the contribution of each component of the flux. The neutron portion of the second hump is only significant between $0.2 \mathrm{GeV} / \mathrm{c}$ and $1 \mathrm{GeV} / \mathrm{c}$, decreasing dramatically as the energy increases. In opposition, the muonic component increases in the same energy range, having its maximum value near $10 \mathrm{GeV} / \mathrm{c}$.

The plot shows two humps for each curve representing the secondary particle flux. The first hump represents the electromagnetic component (electrons, positrons and photons), while the second, made up of two smaller humps, represents the flux of neutrons and muons, respectively. 


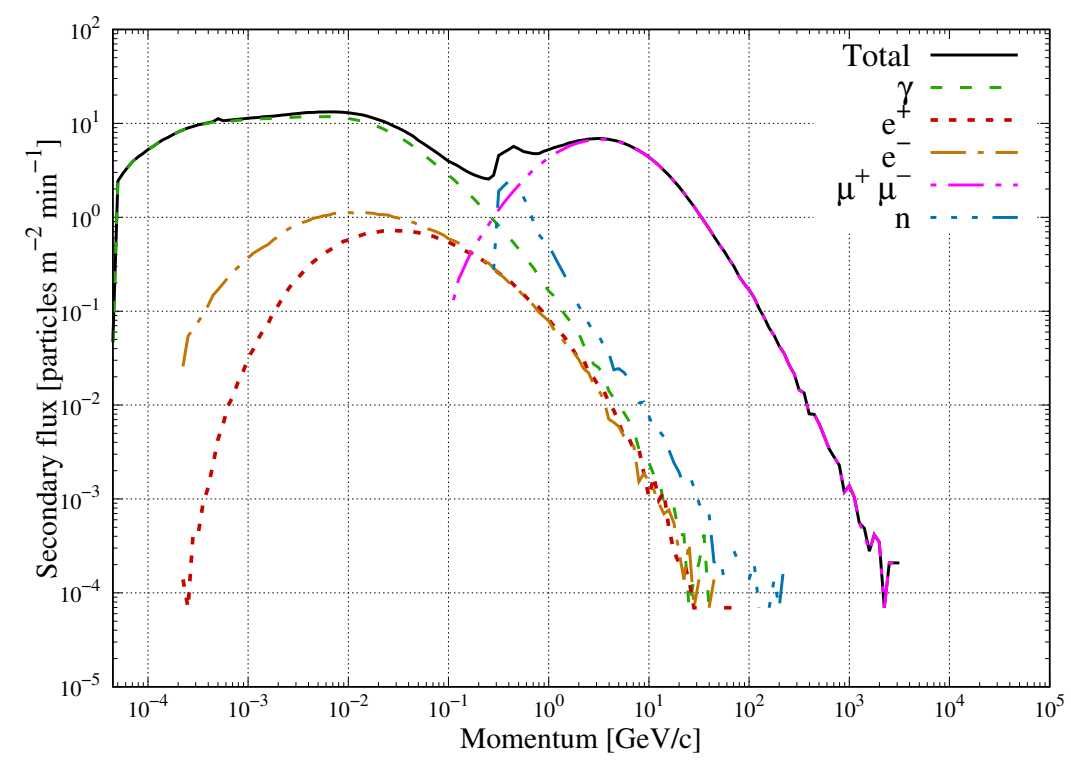

Figure 3: Simulation of the energy spectrum of secondaries, at the level of Bucaramanga, using the atmospheric profile of April. The solid line represents the total spectrum of secondary and the dashed lines represents the contribution of photons, electrons, positrons, muons, neutrons and protons separately.

We ran a total of 12 flux simulations [2] using a GDAS monthly atmospheric profile and, finally, one simulation using the CORSIKA mid-latitude summer predefined profile available in the ATMEXT configuration.

Figure 4 shows the total secondary flux as a function of energy at the altitude of Bucaramanga, using different atmospheric models. The solid line corresponds to the simulation using the default Sub-tropical Summer predefined profile, and the dashed lines correspond to the 12 monthly GDAS atmospheric models. The two solid lines surrounding the dashed lines correspond to November and April and represent the months with the highest and lowest flux. As a preliminary result, we observed a higher flux with the Sub-tropical Summer profile compared to the 12 monthly profiles, with the most significant difference in April.

We observed some differences between April and November, the latter being the closest to the sub-tropical summer model.

\section{Final remarks}

We have devised a methodology that enables one to obtain a month-by-month averaged atmospheric profile for any geographic location. This methodology, implemented using the GDASTOOL code, extracts meteorological data for Bucaramanga in two different hours of the day: 0:00h and 12:00 (UTC-5 local time), during a whole year. In this way, we have created 12 atmospheric profiles for the year 2018 and compare them with predefined atmospheric profiles available in CORSIKA. We observed significant differences in the flux of particles measured at ground level.

It is essential to clarify that monthly atmospheric profiles over a year are not sufficient to represent the average climatic variability in the tropics. We need atmospheres that cover a more significant number of years. Thus, the observed differences, rather than being definitive percentages, 


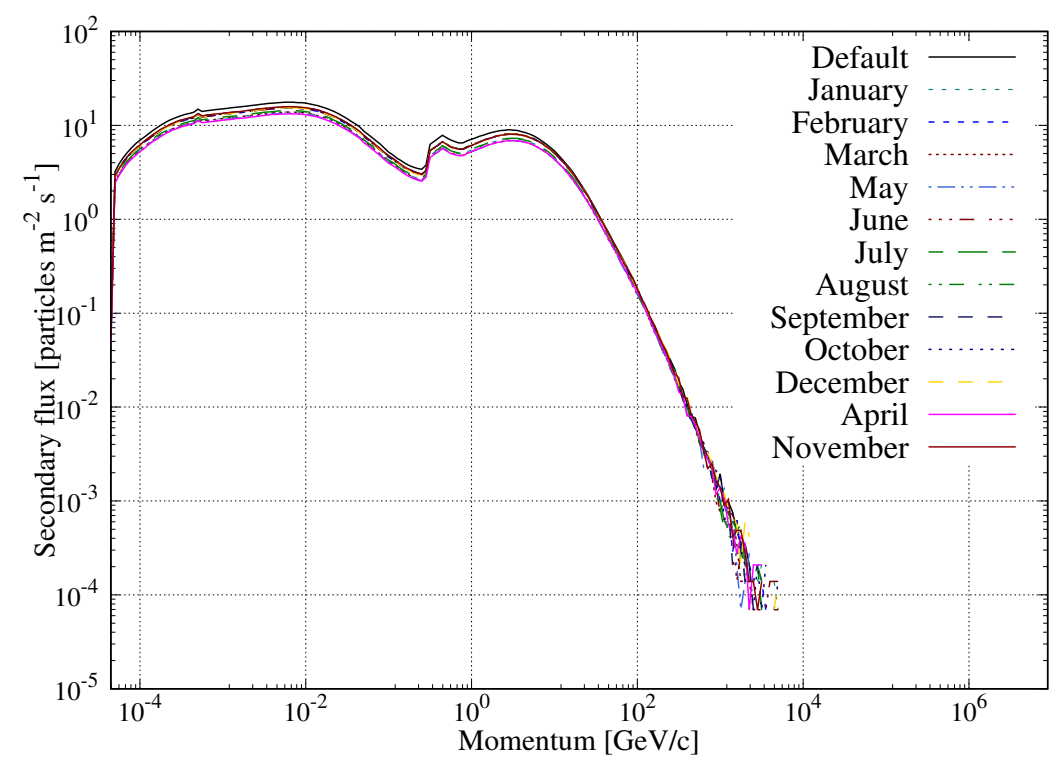

Figure 4: Simulation of total secondary flux as a function of energy at the height of Bucaramanga, using different means of interaction: The solid line represents the default Sub-tropical Summer profile, and the dashed lines correspond to the 12 monthly atmospheric profiles. The estimations show a higher flux with the Sub-tropical Summer profile, compared to the 12 monthly atmospheric profiles.

suggest the importance of continuing to study these effects in greater detail-for example, long-term climatic events such as the El Niño and La Niña phenomena.

\section{Acknowledgements}

Simulations presented in this paper were carried out using the GridUIS-2 experimental testbed, being developed under the Universidad Industrial de Santander (SC3UIS) High Performance and Scientific Computing Centre, development action with support from UIS Vicerrectoria de Investigación y Extension (VIE-UIS) and several UIS research groups as well as other funding bodies (http://www. sc3 . uis. edu.co).

The LAGO Collaboration is very thankful to the Pierre Auger Collaboration for their continuous support. It is also acknowledged the support of the LAGO Collaboration members.

\section{References}

[1] Suárez-Durán M. Variaciones del flujo de radiación cósmica en distintos escenarios geofísicos. Phd thesis, School of Physics, Universidad Industrial de Santander, Bucaramanga, Colombia, 2019.

[2] Sarmiento-Cano C, Suárez-Durán M et al. Performance of the LAGO water Cherenkov detectors to cosmic ray flux. arXiv:2010.14591v1 [astro-ph.IM] 27 Oct 2020.

[3] National Center for Environmental Prediction (NCEP). Global data assimilation system (gdas1). Technical report, NOAA - National Oceanic and Atmospheric Administration. ftp : //arlftp.arlhq. noaa.gov/pub/archives/gdas1/ Last accessed at 2019-07-8. 
[4] D. Heck, J. Knapp, et al. Corsika: A monte carlo code to simulate extensive air showers. Technical Report FZKA 6019, Forschungszentrum Karlsruhe GmbH, 1998.

[5] M. Spurio. Particles and Astrophysics, A multi-messenger approach. Switzerland, Springer, 2015.

[6] Abreu P, Aglietta M, Ahlers M et al. Description of atmospheric conditions at the pierre auger observatory using the global data assimilation system (gdas). Astroparticle Physics 2012; 35(9): 591-607.

[7] Metropolitan Area of Bucaramanga (AMB). Temperature data for the city of bucaramanga. http://racimo.mpsig.com/default/reportes/detallado. 


\section{Full Authors List: LAGO Collaboration}

V. Agosín ${ }^{20}$, A. Alberto ${ }^{3}$, C. Alvarez ${ }^{16}$, J. Araya ${ }^{20}$, R. Arceo $^{16}$, O. Areso ${ }^{13}$, L. H. Arnaldi ${ }^{2}$, H. Asorey ${ }^{14,7}$, M. Audelo ${ }^{9}$, M.G. Ballina-Escobar $^{19}$, D. C. Becerra-Villamizar ${ }^{18}$, X. Bertou ${ }^{2}$, K.S. Caballero-Mora ${ }^{16}$, R. Caiza ${ }^{8}$, R. Calderón-Ardila ${ }^{14}$, Calle, J. ${ }^{24}$, A. C. Fauth $^{27}$, E. Carrera Jarrin ${ }^{26}$, L. E. Castillo Delacroix ${ }^{11}$, C. Castromonte ${ }^{25}$, Cazar-Ramírez D. ${ }^{26}$, Diego Cogollo ${ }^{28}$, D. A. Coloma Borja ${ }^{26}$, R. Conde ${ }^{1}$, J. Cotzomi ${ }^{1}$, D. Dallara ${ }^{11}$, S. Dasso ${ }^{13,5,6}$, R. Aguiar $^{27}$, Albuquerque, A. ${ }^{28}$, J.H.A.P.Reis ${ }^{27}$, H. De León ${ }^{16}$, R. deLeónBarrios $^{23}$, D. Domínguez ${ }^{8}$, M. Echiburu ${ }^{21}$, M. González ${ }^{2}$, M. Gómez Berisso ${ }^{2}$, J. Grisales-Casadiegos ${ }^{23}$, A. M. Gulisano ${ }^{13,12,6}$, Juan Carlos Helo ${ }^{17}$, Condori, C. A. H. ${ }^{24}$, J. E. Ise ${ }^{11}$, Nascimento, G. K. M ${ }^{28}$, M. A. Leigui de Oliveira ${ }^{29}$, F. L. Miletto ${ }^{27}$, V. P. Luzio ${ }^{29}$, F. Machado $^{25}$, Juan F. Mancilla-Caceres ${ }^{22}$, D. Manriquez ${ }^{20}$, A. Martínez-Méndez ${ }^{23}$, O. Martinez ${ }^{1}$, R. Mayo-García ${ }^{3}$, L.G. Mijangos ${ }^{22}$, Miranda, $\mathrm{P}^{24}$, M. G. Molina ${ }^{11}$, I.R. Morales ${ }^{19}$, O.G Morales-Olivares ${ }^{16}$, E. Moreno-Barbosa ${ }^{1}$, P. Muñoz ${ }^{17}$, Nina, C ${ }^{24}$, L.A. Núñez ${ }^{23}$, L. Otininano ${ }^{4}$, R. Pagán-Muñoz ${ }^{3}$, K. M. Parada-Jaime ${ }^{18}$, H. M. Parada-Villamizar ${ }^{18}$, R. Parra $^{10}$, J. Peña-Rodríguez ${ }^{23}$, M. Pereira ${ }^{13}$, Y. A. Perez-Cuevas ${ }^{18}$, H. Perez ${ }^{19}$, J. Pisco-Guabave ${ }^{23}$, Raljevic, M. $^{24}$, M. Ramelli ${ }^{13}$, C. Ramírez ${ }^{22}$, Rivera, $\mathrm{H}^{24}$, L. T. Rubinstein ${ }^{13}$, A.J. Rubio-Montero $^{3}$, J.R. Sacahui ${ }^{19}$, H. Salazar ${ }^{1}$, N. Salomón ${ }^{11}$, J. Samanes ${ }^{4}$, N.A. Santos ${ }^{5}$, C. Sarmiento-Cano ${ }^{14}$, I. Sidelnik ${ }^{2}$, Mayra B. Silva $^{22}$, O. Soto ${ }^{17}$, M. Suárez-Durán ${ }^{18,31}$, Subieta Vasquez, M. ${ }^{24}$, Terrazas C. ${ }^{24}$, Ticona, R. ${ }^{24}$, T. Torres Peralta ${ }^{11}$, Pablo A. Ulloa ${ }^{17}$, Z.R. Urrutia ${ }^{22}$, N. Vásquez ${ }^{8}$, A. Vázquez-Ramírez ${ }^{23}$, A. Vega ${ }^{20}$, P. Vega ${ }^{17}$, J. Vega $^{4}$, A. Vesga-Ramirez ${ }^{14}$, D. Vitoreti ${ }^{30}$, R. Wiklich Sobrinho ${ }^{29}$,

${ }^{1}$ Benemérita Universidad Autónoma de Puebla". ${ }^{2}$ Centro Atómico Bariloche (CNEA/CONICET/IB)". ${ }^{3}$ CIEMAT". ${ }^{4}$ Comisión Nacional de Investigación y Desarrollo Aeroespacial". ${ }^{5}$ Departamento de Ciencias de la Atmósfera y los Océanos, Facultad de Ciencias Exactas y Naturales, Universidad de Buenos Aires.". ${ }^{6}$ Departamento de Física (FCEN,UBA)". ${ }^{7}$ Departamento Física Médica, CNEA-CONICET-UNSAM". ${ }^{8}$ Escuela Politécnica Nacional". ${ }^{9}$ Escuela Superior Politécnica de Chimborazo". ${ }^{10}$ European Soutern Observatory (ESO)". ${ }^{11}$ Facultad de Ciencias Exactas y Tecnología (FACET) - Universidad Nacional de Tucumán (UNT)". ${ }^{12}$ Instituto Antártico Argentino, Dirección Nacional del Antartico, Instituto de Astronomía y Física del Espacio (UBA-CONICET)". ${ }^{13}$ Instituto de Astronomía y Física del Espacio, IAFE (UBA-CONICET)". ${ }^{14}$ Instituto de Tecnologías en Detección y Astropartículas (CNEA, CONICET,UNSAM)". ${ }^{16}$ Universidad Autónoma de Chiapas". ${ }^{17}$ Universidad de La Serena". ${ }^{18}$ Universidad de Pamplona". ${ }^{19}$ Universidad de San Carlos". ${ }^{20}$ Universidad de Valparaíso". ${ }^{21}$ Universidad de Viña del Mar". ${ }^{22}$ Universidad del Valle de Guatemala". ${ }^{23}$ Universidad Industrial de Santander". ${ }^{24}$ Universidad Mayor de San Andrés". ${ }^{25}$ Universidad Nacional de Ingeniería". ${ }^{26}$ Universidad San Francisco de Quito". ${ }^{27}$ Universidade Estadual de Campinas". ${ }^{28}$ Universidade Federal de Campina Grande". ${ }^{29}$ Universidade Federal do ABC". ${ }^{30}$ Universidade Federal do Recôncavo da Bahia". ${ }^{31}$ Université Libre de Bruxelles, Brussels, Belgium.". 